\title{
Pansinusitis aguda odontogénica en adolescente: Reporte de caso.
}

\section{Acute odontogenic pansinusitis in adolescent: Case report.}

\author{
Hans Montanares ${ }^{1 * 2,3}$, Carmen Gloria li $^{1}$, Priscilla Brebi ${ }^{1}$, Carlos Manterola ${ }^{2}$, David Díaz ${ }^{3}$ \\ 1. Universidad de La Frontera, Laboratorio de \\ Patología Molecular, CEMT-BIOREN, Temuco, \\ Chile \\ 2. Universidad de La Frontera, Departamento de \\ Cirugía y CEMyQ, Temuco, Chile \\ 3. Hospital Abraham Godoy Peña. Ciudad Lautaro, \\ Chile \\ * Correspondencia autor. Hans Montanares \\ Dirección: Av. Alemania 0458 Temuco | Teléfono: \\ 45-2596588 | E-mail: hans.montanares@gmail.com \\ Trabajo recibido el 28/01/2018. \\ Aprobado para su publicación el 13/10/2018

\begin{abstract}
RESUMEN
La Pansinusitis aguda odontogénica es un cuadro infeccioso infrecuente que afecta a todos los senos paranasales, en el cual se hace necesario un diagnóstico precoz para obtener una menor morbilidad. Un correcto diagnóstico requiere una evaluación dental exhaustiva, apoyándose con imágenes apropiadas. El presente caso clínico reporta paciente femenino de 17 años, sin enfermedades crónicas de base, diagnosticada con pansinusitis aguda odontogénica a través de examen de tomografía axial computarizada. Fue manejada de forma intrahospitalaria con un equipo multidisciplinario para su recuperación. Tratada con antibioterapia de amplio espectro vía parenteral y manejo del dolor, posterior al alta médica
\end{abstract} \\ se realizó endodoncia del diente afectado y rehabilitación con prótesis fija unitaria.
}

PALABRAS CLAVE:

Pansinusitis, Sinusitis odontógenas, Seno maxilar.

Rev. Clin. Periodoncia Implantol. Rehabil. Oral Vol. 12(2); 90-92, 2019.

\begin{abstract}
Acute Odontogenic Pansinusitis is an infrequent infectious disease that affects all the paranasal sinuses. It requires an early diagnosis to obtain a lower morbidity. A correct diagnosis requires thorough dental evaluation, supported by appropriate images. The present clinical case reports a 17-year-old female patient, without chronic underlying diseases, with anacute Odontogenic Pansinusitis diagnosed through a computerized axial tomography scan. She was treated by a multidisciplinary team for recovery, through parenteral wide spectrum antibiotic therapy and pain management. After medical discharge, root canal of the affected tooth and rehabilitation with fixed unitary prosthesis were performed.

KEY WORDS:

Pansinusitis, Odontogenic sinusitis, Maxillary sinus.
\end{abstract}

Rev. Clin. Periodoncia Implantol. Rehabil. Oral Vol. 12(2); 90-92, 2019

\section{INTRODUCCIÓN}

En las infecciones piogénicas del territorio maxilofacial, el origen dental cumple un rol trascendental, generando una enfermedad grave debido a los potenciales daños a la zona orbitaria e intracraneal ${ }^{(1)}$. Es así como existe evidencia que entre $10 \%$ y $12 \%$ de las infecciones del seno maxilar son infecciones odontógenas ${ }^{(2,3)}$. La pansinusitis es un cuadro infrecuente cuando la evaluación maxilofacial se centra en la etiología odontogénica ${ }^{(1)}$, en Chile no existen estudios de prevalencia para este cuadro agudo con origen dental; sin embargo, el diagnóstico precoz, se asocia a menor morbilidad y mortalidad(4).

El diagnóstico de las sinusitis odontógenas requiere de una evaluación dental exhaustiva que incluya exámenes imagenológicos apropiados. La causa más común de estas patologías son los abscesos dentales, la enfermedad periodontal que ha perforado la membrana de Schneider, irritación e infección secundaria causada por cuerpos extraños intra antrales,y perforaciones sinusales durante una exodoncia ${ }^{(2)}$.

La sinusitis odontogénica se reporta con mayor frecuencia en la cuarta década de la vida, siendo más prevalente en mujeres. La iatrogenia $(55,97 \%)$ es la primera causa, seguido por periodontitis $(40,38 \%)$ y quistes odontógenos $(6,66 \%)^{(5)}$. El origen es de tipo polimicrobiano ${ }^{(6)}$ por lo que su tratamiento incluye uso de antimicrobianos contra los patógenos mixtos de la microbiota oral(2).

El propósito de este reporte es presentar un caso de una paciente de 17 años diagnosticada con pansinusitis aguda (PA) odontogénica, en el cual se discuten características clínicas y tratamiento realizado, resaltando el diagnóstico precoz y trabajo multidisciplinario.

\section{INFORME DEL CASO}

El siguiente reporte de caso contó con el asentimiento de la paciente y consentimiento informado del tutor legal.

Paciente de sexo femenino de 17 años, que acude a la consulta dental por intenso dolor en diente 2.2 (incisivo lateral izquierdo) y aumento de volumen de hemiarcada izquierda. La paciente relata en la anamnesis que está en tratamiento de endodoncia y recibiendo paracetamol de 500 mg cada 6 horas, pero presenta mala respuesta a analgesia. Al examen clínico se observa diente 2.2 trepanado con conducto radicular expuesto a cavidad bucal, aumento de volumen en fondo de vestíbulo, dolor a la percusión y trismus. Al momento de la consulta, la paciente presentó presión arterial de 129/70 mm Hg, frecuencia cardíaca de 97 por minuto, saturación de $99 \%$ y temperatura axilar de $37,9^{\circ} \mathrm{C}$.

Se indicó una radiografía periapical del diente afectado, con la que se evidenció un área radiolúcida apical y para apical mesial de límites netos y forma redondeada (Figura 1).

Se administró anestesia local con lidocaína y epinefrina 1:100.000, sin lograr mejoría sintomática, se realizó drenaje de absceso por conducto dental e irrigación intraconducto con clorhexidina al $2 \%$ y se selló el 


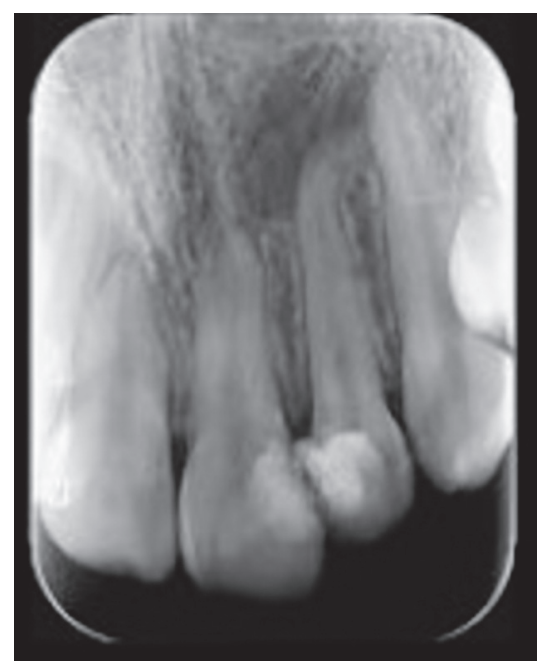

Figura 1. Radiografía periapical. Diente 2.2 inicial.

acceso con óxido de zinc-eugenol. Se decidió derivar inmediatamente a un centro hospitalario ante posibilidad de cuadro séptico, realizar estudios complementarios y evaluación por endodoncia.

En el centro hospitalario se verifica valor de proteína $C$ reactiva (PCR), de 36,1 $\mathrm{mg} / \mathrm{L}$ (valor de referencia 0 - 10). El hemograma evidenció parámetros normales, a excepción de la VHS (Velocidad de sedimentación eritrocitaria) que presentó $38 \mathrm{~mm} / \mathrm{hr}$ (valor de referencia 1 - 20), lo cual se relacionaría con el proceso infeccioso en curso, no se observó variaciones en la cantidad de leucocitos totales, ni en los segmentados.

Se evaluó la función renal mediante la determinación de creatinina, nitrógeno ureico y velocidad de filtrado glomerular previa a la administración de los medicamentos, lo cual resultó normal.

La tomografía axial (TAC) máxilo facial muestra velamiento de todas las cavidades perinasales con niveles hidro-aéreos de senos maxilares y senos esfenoidal (Figuras 2, 3 y 4), confirmando el diagnóstico imagenológico de PA.

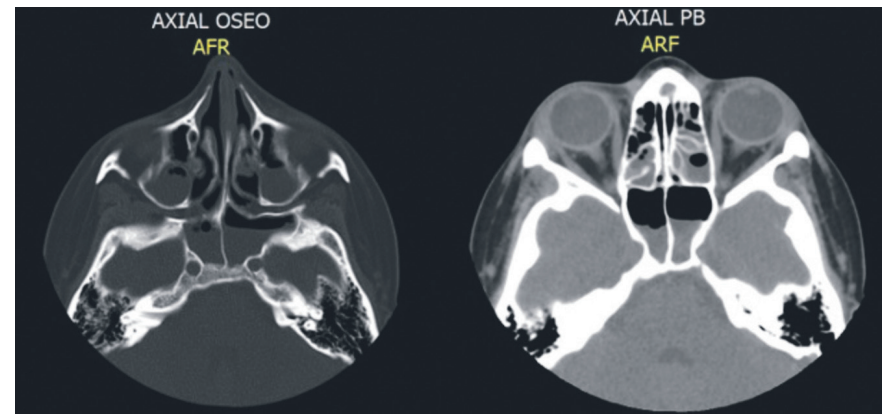

Figura 2. Tomografía Axial Computarizada. Corte axial óseo (izquierda) y corte axial de tejidos blandos (derecha).

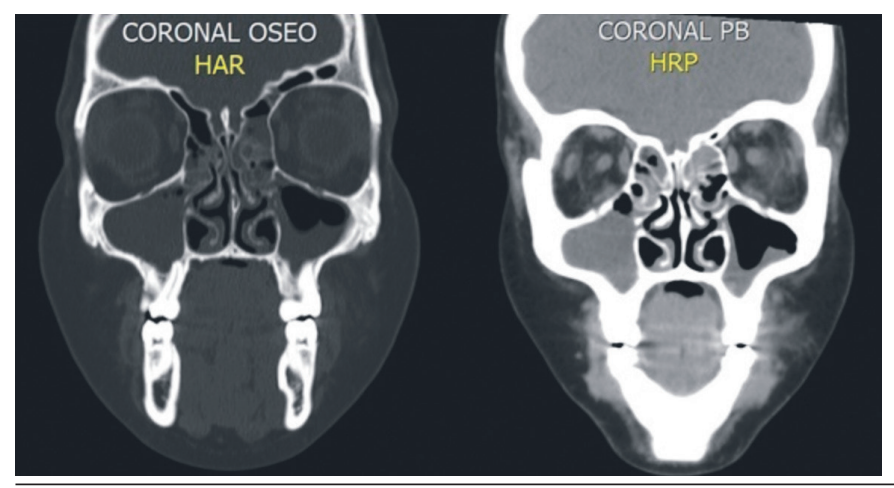

Figura 3. Tomografía Axial Computarizada. Corte coronal óseo (izquierda) y corte coronal de tejidos blandos (derecha).

Tras la evaluación de especialistas en medicina interna y otorrinolaringología, se decidió hospitalizar con antibióticoterapia endovenosa, se administró ceftriaxona 2 gr diarios, asociado a

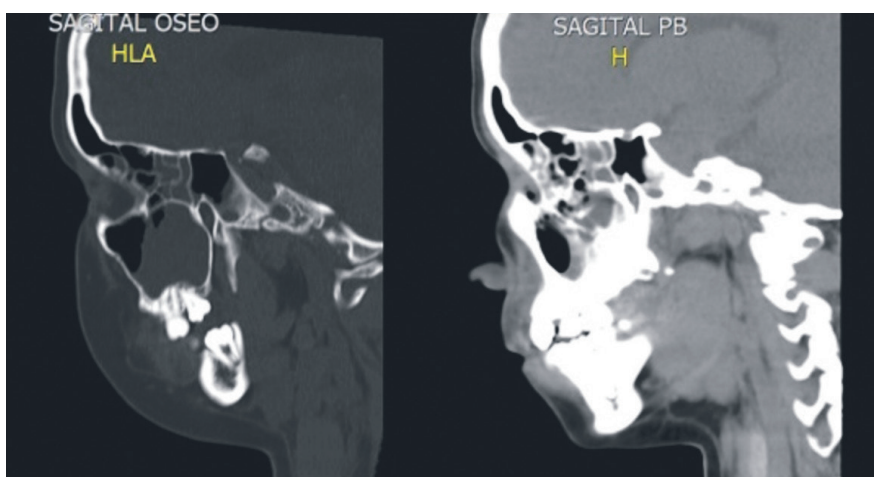

Figura 4. Tomografía Axial Computarizada. Corte sagital óseo (izquierda) y corte sagital de tejidos blandos (derecha).

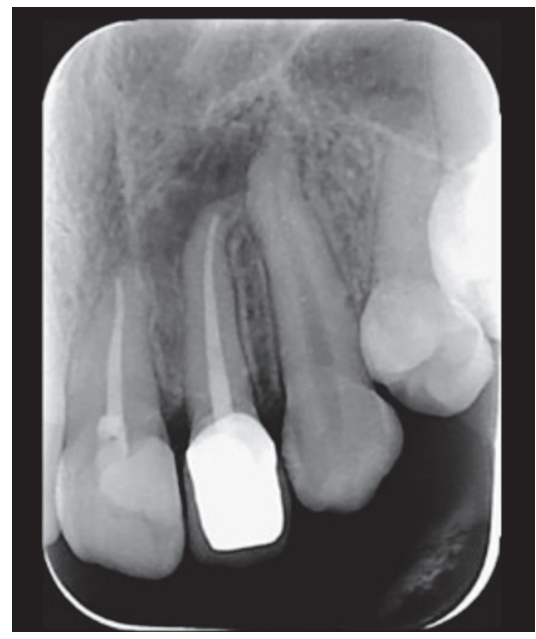

Figura 5. Radiografía periapical. diente 2.2, un mes después de finalizado el tratamiento

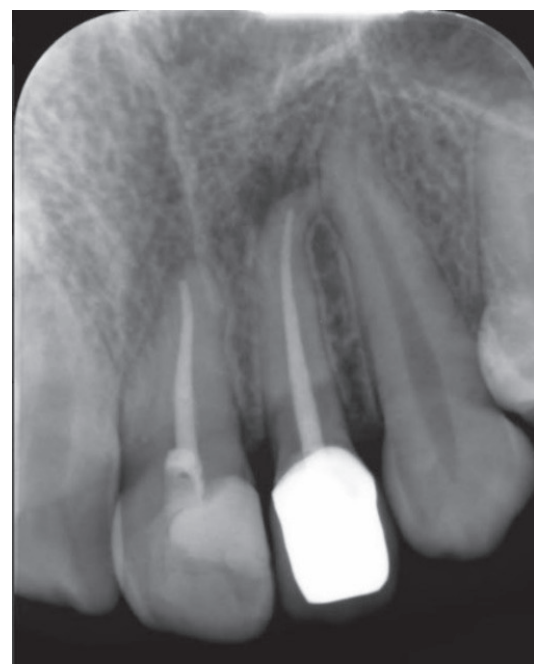

Figura 6. Radiografía periapical. Diente 2.2, seis meses después de finalizado el tratamiento.

clindamicina $600 \mathrm{mg}$ cada 8 horas por 7 días; el manejo analgésico fue con ketoprofeno $100 \mathrm{mg}$ cada 8 horas y ketorolaco $30 \mathrm{mg}$ por 5 días (ambos vía oral). Posteriormente, se mantuvo con Paracetamol $500 \mathrm{mg}$ cada 8 horas por vía oral.

La paciente estuvo hospitalizada por 7 días, evolucionando favorablemente. Requirió evaluación post-tratamiento de la función hepática, por las altas dosis de fármacos, la cual resultó normal. Se evaluó la función renal, la cual presentó normalidad. El nitrógeno ureico y electrolitos post-tratamiento se encontraron igualmente normales y el valor de PCR pre-alta fue de $6,8 \mathrm{mg} / \mathrm{L}$.

Luego se practicó endodoncia en diente 2.2 y diente 2.1 (incisivo central izquierdo); se realizó prótesis fija unitaria en diente 2.2 y se controló 
radiográficamente a un mes de finalizado el tratamiento endodóncico apreciándose una disminución de la zona radiolúcida (Figura 5).

Al sexto mes posterior al tratamiento, paciente refiere no tener molestia alguna. Se decide controlar con radiografía periapical de diente 2.2, con la que se verifica una disminución notable del área radiolúcida (Figura 6).

\section{DISCUSIÓN}

Realizar una endodoncia sin un correcto manejo microbiológico de la zona afectada en un diente con sospecha de raíz antral tiene un alto riesgo de producir sinusitis maxilar. Existe evidencia que apoya el hecho que la edad más frecuente en que se producen cuadros de sinusitis maxilar odontogénica es en la cuarta década ${ }^{(5)}$; $\sin$ embargo, este reporte presenta el caso de un adolescente y en un diente que comúnmente no posee raíz antral, por lo que se advierte siempre una prudencia en el tratamiento de dientes con variaciones anatómicas radiculares.

La literatura describe principios para el tratamiento de infecciones odontogénicas, los investigadores Velasco y Soto(7) adaptaron el protocolo de Peterson y col. . $^{(8)}$ resumiéndolos en: (i) determinar la etiología y severidad de la infección, (ii) evaluar antecedentes médicos y sistema inmune del paciente, (iii) decidir lugar de atención, (iv) tratamiento quirúrgico, (v) soporte médico y nutricional, (vi) elección y prescripción de antibioterapia adecuada y (vii) evaluación periódica del paciente.

La microbiología de la sinusitis de origen odontogénica es polimicrobiana, Brook reportó una tasa del 10\% de microorganismos aerobios, $50 \%$ de anaerobios y $40 \%$ mixto (aerobios y anaerobios), los microorganismos aerobios que predominaron en su estudio fueron: Streptococcus a hemolítico, Streptococcus microaerofílico, y Staphylococcus aureus. Los microorganismos anaerobios que predominaron fueron: Peptostreptococcus spp. y Fusobacterium spp., Prevotella pigmentada y Porphyromonas spp. 2, microorganismos predominantes en infecciones periodontales y endodónticas ${ }^{(9,10)}$, por lo que la terapia antimicrobiana es la columna vertebral del tratamiento, prefiriendo siempre antibióticos que afecten a los microorganismos responsables.

El flujo constante de microorganismos provenientes de la microbiota oral hacia el conducto abierto y la ulterior infección del tejido periapical, fue la causa del cuadro que indujo la respuesta inmune y la diseminación a espacios aéreos de la región máxilo facial a lo largo de vía de menor resistencia. El aumento de la inflamación de los senos maxilares puede producir una reducción del oxígeno, disminución del flujo sanguíneo en la mucosa y depresión de la acción ciliar, este cambio que se ve reflejado en la disminución del $\mathrm{pH}$ de la zona infectada respalda el crecimiento de los microorganismos anaeróbios estrictos al proporcionar un potencial óptimo de óxido-reducción ${ }^{(3,11)}$

El diagnóstico precoz es fundamental en el pronóstico de la PA odontogénica. Se sospecha el origen dental cuando la aparición de la sinusitis acontece después de un episodio de odontalgia o procedimiento dental ${ }^{(12)}$. El manejo multidisciplinario permite un adecuado tratamiento farmacológico y rehabilitador, de lo contrario, los cuadros pueden evolucionar a complicaciones graves con afectación encefálica y orbitaria $^{(13)}$. Las complicaciones cerebrales por sinusitis presentan baja incidencia; sin embargo, cuando ocurren son de extrema gravedad, pues la propagación de la infección se asocia a mortalidad del paciente ${ }^{(14)}$.

Durante el tratamiento es necesaria una estricta vigilancia, apoyándose en pruebas de laboratorio e imágenes. En el presente tratamiento no se realizó hemocultivo con antibiograma, dada la buena respuesta empírica de los antibióticos suministrados, pero al existir riesgo de resistencia antibiótica se sugiere como parte del tratamiento.

Cuando el origen de la PA es dental, es necesario el control de la evolución del caso, mediante radiografías periapicales y TAC ${ }^{(12)}$; en este caso fue imprescindible la información dada por las imágenes del TAC para observar el velamiento de las cavidades de la región Máxilo facial y su adecuado diagnóstico.

Luego del tratamiento farmacológico es necesario el tratamiento definitivo del foco. El cirujano dentista juega un rol importante en la rehabilitación del diente, por lo que, ante un conducto infectado, se sugiere una endodoncia en los dientes afectados, posterior restauración y prótesis fija unitaria, realizando controles odontológicos periódicos para evitar sobreinfección.

\section{CONCLUSIÓN}

La PA odontogénica presenta baja frecuencia, sin embargo, debe ser correctamente diagnosticada por un equipo multidisciplinario y apoyado con exámenes complementarios. El tratamiento farmacológico de elección es antibióticos por vía parenteral, respaldado con hemocultivo y antibiograma, y analgesia durante la hospitalización. La monitorización de los signos y síntomas es clave para evaluar la fase de recuperación sistémica. La eliminación de la causa con endodoncia, rehabilitación dental y controles odontológicos subsiguientes, disminuyen el riesgo de complicaciones futuras.

\section{CONFLICTO DE INTERÉS}

Los autores declaran no presentar conflicto de interés.

\section{RELEVANCIA CLÍNICA}

La Pansinusitis aguda odontogénica presenta baja frecuencia sin embargo, debe ser correctamente diagnosticada por un equipo multidisciplinario y apoyado con exámenes complementarios. El tratamiento farmacológico de elección es antibióticos por vía parenteral, respaldado con hemocultivo y antibiograma y analgesia durante la hospitalización. La monitorización de los signos y síntomas es clave para evaluar la fase de recuperación sistémica. La eliminación de la causa con endodoncia, rehabilitación dental y controles odontológicos subsiguientes, disminuyen el riesgo de complicaciones futuras.

\section{Bibliografía}

1. Eufinger $\mathrm{H}$, Machtens $\mathrm{E}$. Purulent pansinusitis, orbital cellulitis and rhinogenic intracranial complications. J Craniomaxillofac Surg. 2001;29(2): 111-117.

2. Brook I. Microbiology of acute sinusitis of odontogenic origin presenting with periorbital cellulitis in children. Ann Otol Rhinol Laryngol. 2007;116(5): 386-388.

3. Fernandez M, Gonzalez P, Mardones M, Bravo R. Complicaciones severas de las infecciones odontogenicas. Rev Med Clin Condes 2014;25(3): 529-533.

4. Poghosyan A, Fanarjyan R, Nersisyan V, Harutyunyan G, Zakaryan A. Odontogenic pansinusitis complicated by orbital cellulitis, epidural empyema and intracerebral abscess of fronto-basal region : Case Report. J Interdiscipl Med Dent. 2014;2(6): 2-8. 5. Arias O, Barona C, Santos JA, Martínez N, Martínez JM. Meta-analisis of the etiology of odontogenic maxillary sinusitis. Med Oral Patol Oral Cir Bucal. 2010:15(1):3-6. 6. Nash D, Wald E. Sinusitis. Pediatr Rev. 2001;22(4):111-117.

7. Velasco I, Soto R. Principios para el tratamiento de infecciones Odontogénicas con distintos niveles de complejidad. Rev Chil Cir. 2012;64(6):586-598.

8. Peterson LJ. Contemporary management of deep infections of the neck. J Oral Maxillofac Surg. 1993;51:226-31.
9. Brook I. Microbiology and management of endodontic infections in children. J Clin Pediatr Dent. 2003;28:13-7

10. Brook I, Frazier EH, Gher ME. Aerobic and anaerobic microbiology of periapical abscess. Oral Microbiol Immunol. 1991;6:123-5.

11. Brook I. Sinusitis of odontogenic origin. Review article. Otolaryngol Head Neck Surg. 2006;135:349-355.

12. Rubio J, García J, Gutiérrez J, Hueto J. Chávez M, Ferrés E. Pansinusitis y afectación intracraneal por implante dental. Rev Esp Cir Oral Maxilofac. 2012;34(1): 35-37

13. Wysluch A, Maurer P, Ast J, Kunkel K, Bochum, Minden. Orbital complications due to an acute odontogenic focus in a child. A case report. Oral Surg Oral Med Oral Patho Oral Radiol Endod. 2009;107:e39-42.

14. Bayonne E, Kania R, Tran P, Huy B, Herman P. Intracranial complications of rhinosinusitis. A review, typical imaging data and algorithm of management. Rhinology. 2009;47:59-65. 